\title{
Effect of Detergents on the $\boldsymbol{N}$ - and Ring-Hydroxylation of 2-Acetamidofluorene by Hamster Liver Microsomal Preparations
}

\author{
By PRABHAKAR D. LOTLIKAR and ELLEN N. DWYER \\ Fels Research Institute and the Department of Biochemistry, \\ Temple University School of Medicine, Philadelphia, PA 19140, U.S.A.
}

(Received 30 September 1976)

\begin{abstract}
Effects of detergents such as cholate, deoxycholate and Triton X-100 were studied on $N$ and ring-hydroxylation of 2-acetamidofluorene by reconstituted and unresolved microsomal systems from livers of hamsters pretreated with 3-methylcholanthrene. Triton $\mathrm{X}-100(2.5 \mathrm{mg} / \mathrm{nmol}$ of cytochrome $P-448)$ inhibited $N$ - and ring-hydroxylation by whole microsomal preparations by 40 and $90 \%$ respectively. Deoxycholate at the same concentration inhibited both hydroxylations completely, whereas cholate inhibited $N$ - and ring-hydroxylation by 40 and $50 \%$ respectively. In reconstitution studies, the presence of Triton X-100 $(0.5-1.0 \mathrm{mg} / \mathrm{nmol}$ of cytochrome $P-448)$ along with unsolubilized cytochrome $P-448$ fraction and solubilized reductase fraction increased $N$-hydroxylation to an appreciable extent compared with ring-hydroxylation. Both cholate and deoxycholate at 0.5 $1.0 \mathrm{mg}$ concentrations had a greater stimulatory effect on ring- than on $N$-hydroxylation. Triton X-100 was better than either cholate or deoxycholate in stimulating $N$-hydroxylation activity in such a reconstituted system.
\end{abstract}

A variety of physiological and foreign compounds is oxidized by the mammalian hepatic mixed-function oxidase enzyme system (Lu et al., 1973). Two components of this enzyme system, cytochrome $P-450$ and NADPH-cytochrome $P-450$ reductase, have been partially purified after solubilization with ionic detergents such as sodium cholate and deoxycholate (Lu et al., 1969, 1973; Lu \& West, 1972) and a nonionic detergent, Triton X-100 (Lotlikar et al., 1974).

In the carcinogenesis by 2-acetamidofluorene and several other aromatic amides and amines, a large number of studies has shown that $N$-hydroxylation is an activation step, whereas ring-hydroxylation is an inactivation phenomenon (Miller, 1970; Weisburger $\&$ Weisburger, 1973). Inhibition studies with CO have indicated that cytochrome $P-450$ is involved in both $\mathrm{N}$ - and ring-hydroxylation of 2-acetamidofluorene (Gutmann \& Bell, 1973; Thorgeirsson et al., 1973; Lotlikar \& Zaleski, 1974). Differences in inhibition of $N$ - and ring-hydroxylation of this carcinogen by $\mathrm{CO}$ have suggested involvement of multiple forms of cytochrome P-450 (Lotlikar \& Zaleski, 1974). Pretreatment of hamsters with 3-methylcholanthrene has been shown to increase hepatic microsomal 2-acetamidofluorene $\quad N$-hydroxylation activity severalfold without appreciably increasing ringhydroxylation activity (Lotlikar et al., 1967).

In the present study we report differential inhibitory effects of Triton X-100, sodium cholate and deoxycholate on $N$ - and ring-hydroxylation of 2-acetamidofluorene by microsomal preparations from livers of 3-methylcholanthrene-pretreated hamsters. We also report stimulatory effects of these detergents on hydroxylation activities by a reconstituted system from such liver preparations.

\section{Materials and Methods}

Sources for some of the chemicals used in the present study were as follows: Triton X-100, horse heart cytochrome $c$ (type III), bacterial proteinase VII and NADPH, Sigma Chemical Co., St. Louis, MO, U.S.A.; sodium deoxycholate, Fisher Scientific Co., Fair Lawn, NJ, U.S.A.; sodium cholate, 2-acetamidofluorene and 2-acetamido $\left[9-{ }^{14} \mathrm{C}\right]$ fluorene (sp. radioactivity $86 \mathrm{mCi} / \mathrm{mmol}$ ), Schwarz-Mann Co., Orangeburg, NY, U.S.A.; 3-methylcholanthrene, Eastman Kodak Co., Rochester, NY, U.S.A. All other chemicals were of reagent grade.

A group of 10-12 adult male Syrian hamsters $(100-150 \mathrm{~g}$ body wt.) was injected intraperitoneally with 3-methylcholanthrene $(100 \mathrm{mg} / \mathrm{kg}$ body wt.) suspended in $1 \mathrm{ml}$ of corn oil $24 \mathrm{~h}$ before death. Preparation of liver microsomal fractions, proteinase treatment and isolation of particles after proteinase treatment were as described previously (Lotlikar $e t$ al., 1974). As in the rat (Alvares et al., 1967), CO difference spectra of reduced microsomal preparations from livers of hamsters after 3-methylcholanthrene pretreatment showed a shift in the absorption maximum of cytochrome $P-450$ from 450 to $448 \mathrm{~nm}$ (Lotlikar et al., 1974). Microsomal particles

Vol. 160 
obtained after proteinase treatment are designated as unsolubilized cytochrome $P-448$ fractions. The amount of cytochrome $P-448$ in such cytochrome $P-448$ fractions as measured by the method of Omura \& Sato (1964) was about $1.4 \mathrm{nmol} / \mathrm{mg}$ of protein. These cytochrome $P-448$ fractions had NADPHcytochrome $c$ reductase activity of less than $5 \mathrm{nmol} /$ min per $\mathrm{mg}$ of protein as measured by the procedure of Masters et al. (1971). Liver microsomal NADPHcytochrome $c$ reductase fraction prepared by the procedure of Lu \& West (1972) showed activity of about $2100 \mathrm{nmol} / \mathrm{min}$ per $\mathrm{mg}$ of protein. Such a reductase preparation did not have any detectable amount of cytochrome $\boldsymbol{P}-\mathbf{4 4 8}$. Protein was determined by the method of Lowry et al. (1951), with bovine serum albumin as standard.

The complete incubation medium for $\mathrm{N}$ - and ringhydroxylation of 2-acetamidofluorene contained $50 \mu \mathrm{mol}$ of potassium phosphate buffer, $\mathrm{pH} 7.8$, $6 \mu \mathrm{mol}$ of NADPH, $100 \mu \mathrm{mol}$ of sucrose, $10 \%(\mathrm{v} / \mathrm{v})$ glycerol, $1.12 \mu \mathrm{mol}$ of 2 -acetamidofluorene containing $0.1 \mu \mathrm{Ci}$ of 2 -acetamido[9-14 $\mathrm{C}$ ]fiuorene dissolved in $0.05 \mathrm{ml}$ of methanol, and microsomal protein containing $2 \mathrm{nmol}$ of cytochrome $P-448$ in a total volume of $3.0 \mathrm{ml}$. It is known that glycerol stabilizes detergenttreated cytochrome $P-450$ (Ichikawa \& Yamano, 1967). In the present studies, glycerol was added to the incubation medium as a protective agent for cytochrome $P$-448. Microsomal fractions were prepared and suspended in $0.25 \mathrm{M}$-sucrose; sucrose was therefore added to the incubation medium along with the microsomal fraction. Addition of sucrose did not have any effect on the hydroxylation of 2-acetamidofluorene by liver microsomal preparations. Where indicated, cytochrome $P-448$ fraction containing 2 nmol of cytochrome $P-448$, reductase fraction with NADPH-cytochrome $c$ reductase activity of $600 \mathrm{nmol} / \mathrm{min}, 300 \mu \mathrm{mol}$ of $\mathrm{KF}$ and various amounts of Triton $\mathrm{X}-100$, sodium cholate or sodium deoxycholate were also added to the incubation medium. Where indicated, fluoride was added because the presence of $\mathrm{KF}(0.1 \mathrm{M})$ in the incubation medium was shown to inhibit hamster liver microsomal deacetylase activity and to increase the formation of $\mathrm{N}$-hydroxy-2-acetamidofluorene (Lotlikar et al., 1967). Duplicate samples were preincubated for $30 \mathrm{~min}$ at $5^{\circ} \mathrm{C}$ before addition of 2 acetamidofluorene and NADPH. In the absence of any detergent, such a preincubation did not have any effect on the $\mathrm{N}$ - and ring-hydroxylation of 2acetamidofluorene by either the whole microsomal preparations or a reconstituted system. Such a preincubation was required in order to observe the optimum effect of a detergent on the mixed-function oxidase system. After the addition of 2-acetamidofluorene and NADPH, samples were incubated in air for $20 \mathrm{~min}$ at $37^{\circ} \mathrm{C}$. Extraction of hydroxylated metabolites of 2-acetamidofluorene from the incuba- tion medium with diethyl ether, their separation by paper chromatography and determination by radioactivity measurements were as described previously (Lotlikar et al., 1974).

\section{Results and Discussion}

Various detergents such as deoxycholate, cholate, Lubrol WX and Triton X-100 have been used to solubilize and purify further the membrane-bound mixed-function oxidase system of several hepatic microsomal preparations (Lu et al., 1969, 1973; Mitani et al., 1971; Comai \& Gaylor, 1973; Lotlikar et al., 1974). Resolution of this mixed-function oxidase system after solubilization with such detergents has shown that all three fractions, cytochrome $P-450$, NADPH-cytochrome $P-450$ reductase and a lipid fraction, phosphatidylcholine, are required for oxidation of several physiological substrates, such as steroids and fatty acids, and a variety of foreign compounds, including drugs and a carcinogen,

Table 1. Inhibitory effects of detergents on $N$ - and ringhydroxylation of 2-acetamidofluorene by microsomal preparations from livers of 3-methylcholanthrene-pretreated hamsters

In addition to the complete incubation medium, all samples contained $0.1 \mathrm{M}-\mathrm{KF}$ during incubation. Where indicated, detergent concentration is expressed as $\mathrm{mg}$ of detergent added/nmol of cytochrome $P$-448. Other details are described in the Materials and Methods section. In the absence of any detergent, control microsomal preparations formed $19 \pm 1.5$ and $50 \pm 6.0 \mathrm{nmol}$ of $N$ - and ring-hydroxy2-acetamidofluorene/ $20 \mathrm{~min}$ pre nmol of cytochrome $P-448$ respectively. Results of control microsomal preparations and detergent effects on such perparations are given as means \pm S.E.M. of three analyses. Results for detergent effects are expressed as percentages of the control.

\begin{tabular}{ccc}
$\begin{array}{c}\text { Detergent } \\
(\mathrm{mg})\end{array}$ & $\overbrace{}^{N-}$ & \multicolumn{1}{c}{ Ring } \\
$-\quad 100$ & 100 \\
Triton X-100 & $89 \pm 7$ & $49 \pm 3$ \\
0.5 & $74 \pm 5$ & $15 \pm 5$ \\
1.0 & $61 \pm 4$ & $11 \pm 3$ \\
2.5 & & \\
Sodium cholate & $81 \pm 9$ & $86 \pm 10$ \\
0.5 & $78 \pm 4$ & $72 \pm 7$ \\
1.0 & $64 \pm 8$ & $52 \pm 5$ \\
2.5 & & \\
Sodium deoxycholate & $69 \pm 6$ & $51 \pm 4$ \\
0.5 & $48 \pm 5$ & $24 \pm 3$ \\
1.0 & $3 \pm 1$ & $4 \pm 2$ \\
2.5 & &
\end{tabular}

2-Acetamidofluorine hydroxylation (\% of control) 


\section{Table 2. Effect of detergents on 2-acetamidofluorene hydroxylation by microsomal fractions from livers of 3-methylcholanthrene- pretreated hamsters}

Unsolubilized cytochrome $P-448$ fractions were obtained after proteinase treatment of liver microsomal preparations. Other details are described in the Materials and Methods section. Where indicated, cytochrome $P-448$ fraction containing $2 \mathrm{nmol}$ of cytochrome $P-448$ and reductase fraction containing $600 \mathrm{nmol} / \mathrm{min}$ of NADPH-cytochrome $c$ reductase were added. $\mathrm{KF}(0.1 \mathrm{M})$ was not present during incubation in these samples, except when whole liver microsomal preparation was used. Where indicated, detergent concentration is expressed as $\mathrm{mg}$ of detergent added/nmol of cytochrome $P$-448. In the absence of any detergent, whole microsomal preparations (control) formed $19 \pm 1.5$ and $50 \pm 6.0 \mathrm{nmol}$ of $N$ - and ring-hydroxy-2acetamidofluorene/20 min per nmol of cytochrome $P-448$ respectively. Results of whole microsomal preparations are given as means \pm S.E.M. of three analyses. Results of reconstitution studies are averages of three analyses and are expressed as percentages of the control; experimental variations were less than $10 \%$ of the average of three analyses.

Addition of fraction

Cytochro

2-Acetamidofluorene hydroxylation ( $\%$ of control)

$\begin{array}{cc}N- & \text { Ring- } \\ 0.3 & 0.3 \\ 3.2 & 5.5 \\ & \\ 20 & 13 \\ 37 & 14 \\ 19 & 4.8 \\ & \\ 6.8 & 11 \\ 13 & 19 \\ 7.1 & 13 \\ 7.6 & \\ 11 & 14 \\ 9.2 & 17 \\ & 3.6\end{array}$

benzo[a]pyrene (Lu et al., 1969, 1973; Strobel et al., 1970). In addition to solubilizing the mixed-function oxidase system, it has been shown that detergents such as Triton $\mathrm{X}-100$, deoxycholate and cholate could also partially replace the lipid fraction in the hydroxylation of benzo[ $a]$ pyrene by the reconstituted liver microsomal system from rats pretreated with 3-methylcholanthrene (Lu \& West, 1972).

In our previous studies, cytochromes $P-450$ and $P-448$ were partially purified from rat and hamster liver microsomal preparations after proteinase treatment and Triton X-100 solubilization. Such cytochrome $P-440$ and $P-448$ fractions were able to $N$-hydroxylate 2-acetamidofluorene very efficiently in the presence of deoxycholate-solubilized and purified reductase fraction (Lotlikar et al., 1974; Lotlikar \& Zaleski, 1975). However, we were unable to reconstitute deoxycholate- or cholate-solubilized cytochrome $\boldsymbol{P}-450$ or $\boldsymbol{P}-\mathbf{4 4 8}$ fraction under similar conditions (P. D. Lotlikar, unpublished work).

The present study was designed to compare the effects of the detergents Triton X-100, cholate and deoxycholate on $N$ - and ring-hydroxylation of 2-acetanidofluorene by liver microsomal preparations. It was found that addition of these detergents at $0.5-2.5 \mathrm{mg} / \mathrm{nmol}$ of cytochrome $P-448$ to the incuba- tion medium had differential inhibitory effects on $N$ and ring-hydroxylation of 2-acetamidofluorene by liver microsomal systems from hamsters pretreated with 3-methylcholanthrene (Table 1). Thus Triton $\mathrm{X}-100$ at $0.5 \mathrm{mg} / \mathrm{nmol}$ inhibited ring-hydroxylation by about $50 \%$ without appreciably affecting $N$ hydroxylation. Even at $2.5 \mathrm{mg} / \mathrm{nmol}$ Triton X-100 inhibited $N$-hydroxylation by only $40 \%$. Similar results were obtained with cholate with regard to $N$-hydroxylation. However, in contrast with Triton $\mathrm{X}-100$, cholate, even at $2.5 \mathrm{mg} / \mathrm{nmol}$, inhibited ringhydroxylation by only $50 \%$. Of the three detergents tested, cholate as found to be the least potent inhibitor and deoxycholate was the most potent inhibitor of both $N$ - and ring-hydroxylation, It has also been observed that $N$-hydroxylation by liver microsomal preparation from control hamsters is inhibited to a greater extent by Triton X-100 than is that from hamsters pretreated with 3-methylcholanthrene (P. D. Lotlikar, unpublished work). In previous studies, Triton X-100 has been found to be a very potent inhibitor of $N$-demethylation of both ethylmorphine (Lu \& Levin, 1974) and dimethylnitrosamine (P.D. Lotlikar, unpublished work). The present $N$-hydroxylation data appear to be the first example where Triton X-100 has not been found to be a potent 
inhibitor of the microsomal mixed-function oxidase system.

In previous reconstitution studies, dimethylnitrosamine demethylation activity of unsolubilized cytochrome $P-450$ fraction in the presence of solubilized reductase fraction was about $40 \%$ of that obtained with whole liver microsomal preparations from hamsters (Lotlikar et al., 1975). In contrast, in the present study, unsolubilized cytochrome $P$-448 fraction in the presence of reductase fraction yielded only a small amount of $N$ - and ring-hydroxylation activity (Table 2). The presence of $0.5-1 \mathrm{mg}$ of Triton X-100/nmol increased $N$-hydroxylation to an appreciable extent compared with ring-hydroxylation. A higher concentration of Triton X-100 had an inhibitory effect on both types of oxidations. Both sodium cholate and deoxycholate at $0.5-1 \mathrm{mg} / \mathrm{nmol}$ had greater stimulatory effects on ring-hydroxylation than on $\mathrm{N}$-hydroxylation.

In previous reconstitution experiments, where cytochrome $P-448$ was partially purified after proteinase treatment and Triton X-100 solubilization of microsomal preparations, it was found that $N$ hydroxylation activity was completely restored, whereas ring-hydroxylation activity was restored only to about $20-30 \%$ of the activity of whole microsomal preparations (Lotlikar et al., 1974; Lotlikar \& Zaleski, 1975). However, in the present report (Table 2) $N$-hydroxylation activity was restored to about $40 \%$ of the activity of the whole microsomal system; this might be due to the inhibitory effect of Triton X-100 present in the incubation medium (Table 1).

Differential inhibition of $\mathrm{N}$ - and ring-hydroxylation of 2-acetamidofluorene by CO (Lotlikar \& Zaleski, 1974) and by various detergents such as Triton X-100, cholate and deoxycholate (present data, Table 1) suggest possible involvement of multiple forms of cytochrome $P-450$. Similarly, the above data and the differential stimulatory effect on these hydroxylation reactions by these detergents (present data, Table 2) also suggest different localizations of these various cytochromes $P-450$ in the endoplasmic membrane.
This research was supported in part by a research grant (CA-10604) and a grant (CA-12227) to the Fels Research Institute from the National Cancer Institute, U.S. Public Health Service.

\section{References}

Alvares, A. P., Schilling, G., Levin, W. \& Kuntzman, R. (1967) Biochem. Biophys. Res. Commun. 29, 521-526

Comai, K. \& Gaylor, J. L. (1973) J. Biol. Chem. 248, 4947-4955

Gutmann, H. R. \& Bell, P. (1973) Fed.Proc. Fed. Am. Soc. Exp. Biol. 32, 665

Ichikawa, Y. \& Yamano, T. (1967) Biochim. Biophys. Acta 131, 490-497

Lotlikar, P. D. \& Zaleski, K. (1974) Biochem. J. 144, 427-430

Lotlikar, P. D. \& Zaleski, K. (1975) Biochem. J. 150, 561-564

Lotlikar, P. D., Enomoto, M., Miller, J. A. \& Miller, E. C. (1967) Proc. Soc. Exp. Biol. Med. 125, 341-346

Lotlikar, P. D., Luha, L. \& Zaleski, K. (1974) Biochem. Biophys. Res. Commun. 59, 1349-1355

Lotlikar, P. D., Baldy, W. J., Jr. \& Dwyer, E. N. (1975) Biochem. J. 152, 705-708

Lowry, O. H., Rosebrough, N. J., Farr, A. L. \& Randall, R. J. (1951) J. Biol. Chem. 193, 265-275

Lu, A. Y. H. \& Levin, W. (1974) Biochim. Biophys. Acta 344, 205-240

Lu, A. Y. H. \& West, S. (1972) Mol.Pharmacol. 8, 490-500

Lu, A. Y. H., Junk, K. W. \& Coon, M. J. (1969) J. Biol. Chem. 244, 3714-3721

Lu, A. Y. H., Levin, W., West, S., Jacobson, M., Ryan, D., Kuntzman, R. \& Conney, A. H. (1973) Ann. N.Y. Acad. Sci. 212, 156-174

Masters, B. S. S., Baron, J., Taylor, W. E., Isaacson, E. L. \& LoSpalluto, J. (1971) J. Biol. Chem. 246, 4143-4150

Miller, J. A. (1970) Cancer Res. 30, 559-576

Mitani, F., Alvares, A. P., Sassa, S. \& Kappas, A. (1971) Mol. Pharmacol. 7, 280-292

Omura, T. \& Sato, R. (1964) J. Biol. Chem. 239, 2370-2378

Strobel, H. W., Lu, A. Y. H., Heidema, J. \& Coon, M. J. (1970) J. Biol. Chem. 245, 4851-4854

Thorgeirsson, S. S., Jollow, D. J., Sesame, H., Green, J. \& Mitchell, J. R. (1973) Mol. Pharmacol. 9, 398-404

Weisburger, J. H. \& Weisburger, E. K. (1973) Pharmacol. Rev. 25, 1-66 